\title{
FREQUÊNCIA DAS PARASITOSES INTESTINAIS EM ESCOLAS PÚBLICAS DA BAHIA
}

\author{
FREQUENCY OF INTESTINAL PARASITES IN PUBLIC SCHOOLS IN BAHIA
}

\author{
Rafael de Jesus Alves Neto \\ Secretaria da Saúde do Estado da Bahia - SESAB',Faculdade Santo Agostinho de Itabuna², Universidade Estadual de \\ Santa Cruz - UESC ${ }^{3}$
}

\begin{abstract}
The parasites, highlighting the intestinalis were frequently in public students of low aquisity power in developing country like Brazil. The aim of the work was to verify the prevalence of intestinal parasites infections in public students, and help to prevent with an education program. The ludic activities were using in two public schools of Jequie of Bahia-Brazil. The prevalence of intestinal parasites was determinate between 2014 until 2017. The fecal material was concentrated by spontaneous sedimentation. It was examined 352 fecal samples collected from children and teenagers from 7 to 14 of age. The positive results for one or more parasites was $55.97 \%$. The students that had an intestinal infection received medical treatment. The most prevalent parasite was Schistosoma mansoni 79 (22,4\%), following by A. lumbricoides 69 (19,6\%); T. trichiura 45 (12,8\%); Hookworm 28 (8,0\%); H. nana $15(4,3 \%)$ and $E$. vermicularis $09(2,6 \%)$. The highest prevalence of intestinal parasites and commensals protozoan was the Entamoeba coli 59 (16,8\%). Following by E. histolytica/dispar 52 (14,8\%); G. lamblia 45 (12,8\%); Endolimax nana 45 (12,8\%), and lodamoebabutschlii 30 (8,5\%). It is concluded that these results show that although the educations programs, there is still a significant environmental contamination with intestinal parasites in periphery of Jequie revealing the importance of prevalence estimation and health education.
\end{abstract}

Key words: parasitosis, scholarships, prevalence.

\section{Resumo}

As parasitoses intestinais são frequentes em escolares públicos de baixo poder aquisitivo em países em desenvolvimento como o Brasil. Os objetivos dessa pesquisa foram: verificar a prevalência das parasitoses intestinais em estudantes de escolas públicas do município de Jequié-BA, e auxiliar na prevenção com um programa de educação. Foram utilizadas metodologias lúdicas nas oficinas educativas ministradas em duas escolas municipais. Foi utilizado o método de sedimentação espontânea para a análise do material fecal. A prevalência das parasitoses intestinais foi determinada de 2014 a 2017. Foram analisadas 352 amostras de fezes de estudantes de 7 a 14 anos de idade. Os resultados positivos para um ou mais parasitas foi de 55,97\%. As crianças e adolescentes que apresentaram resultado positivo foram encaminhados ao tratamento. $O$ parasita de maior frequência foi o Schistosoma mansoni 79 (22,4\%) seguido pelo A. lumbricoides $69(19,6 \%) e$ T. trichiura 45 (12,8\%); Ancilostomídeos 28 (8,0\%); H. nana 15 (4,3\%); E. vermicularis 09 (2,6\%). Entre os protozoários parasitas e comensais o de maior prevalência foi a Entamoeba coli 59 (16,8\%); E. histolytica/dispar 52 (14,8\%); G. lamblia 45 (12,8\%); Endolimax nana 45 (12,8\%), e lodamoebabutschlii 30 (8,5\%). Conclui-se que, apesar dos programas de prevenção existentes, ainda existe elevado índice de parasitoses intestinais na periferia de Jequié, revelando a importância de estudos de prevalência $e$ educação em saúde.

Palavras chaves: parasitoses, escolares, prevalência. 
Introdução
As enteroparasitoses na infância assumem grande relevância, não apenas pela morbidade que está associada à frequente diarreia crônica, desnutrição e fatores que podem ocasionar déficit físico e mental, mas também óbito ${ }^{1}$. As intervenções educativas podem estimular ações que contribuam para a prevenção deste tipo de infecção. Os métodos lúdicos são uma ferramenta que tornam o agente ativo no processo ${ }^{2}$.

As crianças representam o grupo mais vulnerável à infestação por parasitas intestinais, uma vez que, geralmente, não realizam medidas de higiene pessoal de forma adequada e, frequentemente, se expõem ao solo e à água, que são importantes focos de contaminação ${ }^{3}$.

Mesmo que nos últimos anos o Brasil tenha apresentado melhorias na qualidade de vida de sua população, as parasitoses ainda são endêmicas em várias regiões do país. As parasitoses intestinais são frequentes em escolares públicos de baixo poder aquisitivo em países em desenvolvimento como o Brasil. Ressaltamos a esquistossomose mansônica que ainda é um problema de saúde pública no município de Jequié-BA. No Brasil, o estado da Bahia se encontra entre os de maior frequência da esquistossomose. Assim sendo, ainda são necessários, em paralelo às pesquisas e controle em algumas cidades, melhorar as medidas sanitárias, médicas e educativas que possam auxiliar na diminuição deste problema.

A falta de saneamento básico, higiene pessoal, doméstica e informação sobre a prevenção das doenças parasitárias são fatores preponderantes que favorecem a transmissão dos parasitas. As parasitoses intestinais apresentam maior prevalência em áreas rurais e periféricas das cidades, sendo a habitação e peridomicílio os locais onde oferecem maior risco de contaminação. As crianças em idade escolar acometidas por parasitoses podem apresentar desde sintomas leves até complicações, às vezes irreversíveis ${ }^{4}$.

A Organização Mundial de Saúde (OMS) em 1993, afirmou que "escolares abrigam algumas infeç̧ões por helmintos mais intensas, com efeitos adversos sobre a saúde, o crescimento e o desempenho escolar" e que se torna viável o tratamento em massa de toda população quando os levantamentos escolares indicarem prevalência de enteroparasitoses acima de $50 \%{ }^{5}$ Apesar de alguns avanços nas últimas décadas, várias regiões do Brasil, principalmente o Nordeste, continuam a apresentar índices relevantes de mortalidade causados por doenças diarreicas, sobretudo em crianças pequenas em idade escolar ${ }^{6}$, logo, nota-se ainda a ineficácia das campanhas e programas de atenção à saúde destinada ao combate e erradicação de doenças parasitárias.

No caso da esquistossomose, que a contaminação está ligada ao contato com a água doce, a prevalência é consideravelmente maior entre crianças e jovens. No município de Jequié, isso acontece devido ao fato da presença de rios, represas e lagos com a presença do molusco Biomphalariaglabratacontaminado com o Shistosoma mansoni. Além disso, o calor intenso durante quase todo o ano propicia a procura de banhos de rios, além das poucas opções de lazer para este público alvo.

Considerando as escolas municipais um local ideal para incluir um programa de educação e realização dos exames coprológicos, este estudo teve como público alvo estudantes de escolas públicas. Foi levada em consideração a ausência de informação sobre as doenças parasitárias em escolares do primeiro ao quinto ano do ensino fundamental, justificando o trabalho nesse público ${ }^{7}$.

O objetivo principal deste estudo foi verificar a prevalência das parasitoses intestinais em alunos de escolas da rede pública da cidade de Jequié - BA. Além disso, ministrar oficinas educativas sobre a prevenção das principais parasitoses, encaminhar os parasitados ao

tratamento, proporcionar atividades educacionais e práticas de diagnóstico aos discentes voluntários da Universidade Estadual do Sudoeste da Bahia - UESB que participaram do estudo.

\section{Metodologia}

Trata-se de um estudo epidemiológico, quantitativo e transversal realizado a partir dos dados dessa pesquisa em duas escolas públicas, e domicílios do bairro Jequiezinho no município de Jequié - BA, Brasil.

O município possui uma população de aproximadamente 150.000 habitantes, tem um clima quente na maior parte do ano e conta com 
uma vasta hidrografia. A escolha das duas escolas foi definida tomando-se como critério o baixo padrão socioeconômico, posição geográfica e infraestrutura ao redor das escolas.

No presente trabalho foi realizado um levantamento parasitológico de fezes entre os anos de 2014 a 2017. Foram realizadas reuniões com os pais ou responsáveis nas escolas, com intuito de esclarecimento sobre a profilaxia das parasitoses. Previamente à coleta do material fecal os responsáveis receberam orientações quanto à coleta de fezes. Foi fornecido recipiente com conservante para coleta aos responsáveis que aceitaram e assinaram o termo de Consentimento Livre e Esclarecimento. Outra atividade desenvolvida auxiliando na prevenção das parasitoses foi a visita domiciliar. Tendo essa, o mesmo objetivo de levar informação sobre a prevenção das parasitoses àqueles que não compareceram nas reuniões da escola. As amostras fecais foram devidamente acondicionadas e transportadas, sendo submetidas ao diagnóstico no Laboratório de Microbiologia, Parasitologia e Imunologia da Universidade Estadual do Sudoeste da Bahia UESB, campus de Jequié - BA.

Foram analisadas 352 amostras fecais pelo método qualitativo de sedimentação espontânea, proposto em 1934 por Hoffman e colaboradores ${ }^{8}$, sendo examinadas duas lâminas por paciente. 0 projeto de pesquisa foi aprovadopelo Comitê de
Ética em Pesquisa da Universidade Estadual do Sudoeste da Bahia, Resolução no 196/96 do Conselho Nacional de Saúde, sob o parecer no 201/2009, e não apresenta qualquer tipo de conflito de interesse.

As oficinas educativas foram ministradas por discentes voluntários da UESB, usando métodos lúdicos. Os escolares que estavam parasitados foram encaminhados ao tratamento médico específico.

\section{Resultados e discussões}

A prevalência geral de parasitoses intestinais encontradas nas 352 amostras de fezes analisadas neste estudo foi de $55,97 \%$, isto é, 197 escolares infectados com pelo menos um ou mais parasitos. E 155 (44,03\%) estudantes apresentaram resultados negativos.

As 352 amostras de fezes analisadas e o resultado específico de cada parasito foram distribuídos de acordo com o ano que foi realizado (tabelas 1 e 2). Foram diagnosticadas seis espécies de helmintos, sendo o Schistosoma mansoni $79(22,4 \%)$ o mais prevalente. As outras espécies menos frequentes e apresentadas em ordem decrescente de prevalência foram: Ascaris lumbricoides 69 (19,6\%), Trichuristrichiura 45 (12,8\%), Ancilostomídeos 28 (8,0\%), Hymenolepis nana 15 (4,3\%), e Enterobius vermiculares 9 $(2,6 \%)$.

Tabela 1 - Resultados dos 352 exames de fezes dos estudantes examinados nos anos de 2014 -2017 no município de Jequié - $\mathrm{BA}$, em relação à presença de ovos de helmintos.

\begin{tabular}{|c|c|c|c|c|c|} 
Helmintos & 2014 & 2015 & 2016 & 2017 & TOTAL - N/\% \\
\hline Schistosoma mansoni & 37 & 12 & 22 & 08 & $79(22,4 \%)$ \\
\hline Ascaris lumbricoides & 15 & 22 & 18 & 14 & $69(19,6 \%)$ \\
\hline Trichuristrichiura & 14 & 08 & 11 & 12 & $45(12,8 \%)$ \\
\hline Ancilostomídeos & 12 & 03 & 09 & 04 & $28(8,0 \%)$ \\
\hline Hymenolepis nana & 06 & 02 & 02 & 05 & $15(4,3 \%)$ \\
\hline Enterobiusvermicularis & 03 & 03 & 02 & 01 & $09(2,6 \%)$
\end{tabular}

Foram detectadas cinco espécies de protozoários, sendo a mais prevalente, o comensal, Entamoeba coli 59 (16,8\%). As outras espécies parasitas menos frequentes foram Giardialamblia52 (14,8\%) e complexo
Entamoebahistolytica/dispar52 (14,8\%). Além dos comensais menos frequentes Endolimax nana e lodamoebabutshilii45 (12,8\%). 
Tabela 2 - Resultados dos 352 exames de fezes dos estudantes examinados de 2014 a 2017 no município de Jequié-BA, em relação à presença de cistos de protozoários.

\begin{tabular}{|c|c|c|c|c|c|}
\hline Protozoários & 2014 & 2015 & 2016 & 2017 & TOTAL $-\mathrm{N} / \%$ \\
\hline Entamoeba coli & 24 & 13 & 19 & 03 & $59(16,8 \%)$ \\
\hline E.histolytica/dispar & 16 & 16 & 10 & 10 & $52(14,8 \%)$ \\
\hline Giardia lambia & 14 & 13 & 15 & 03 & $45(12,8 \%)$ \\
\hline Endolimax nana & 25 & 07 & 08 & 05 & $45(12,8 \%)$ \\
\hline Iodamoebabutshilii & 15 & 06 & 09 & 0 & $30(8,5 \%)$
\end{tabular}

As oficinas educativas foram ministradas semanalmente com duração de uma hora dentro das salas de aulas das escolas públicas escolhidas. A grande maioria das crianças e adolescentes mostrou interesse e alto índice de participação.

A análise epidemiológica da situação das doenças enteroparasitárias no Brasil tem uma relação direta com o nível de saneamento básico, hábitos de higiene, condições de moradias e grau de escolaridade das populações das regiões do país. No século passado, os índices de prevalência de parasitoses no Brasil eram muito altos. No início dos anos 2000, ainda foi registrado em um município baiano o índice de $70,7 \%$ de positividade ${ }^{9}$.

Os países em desenvolvimento, como o Brasil, vêm apresentando uma queda progressiva nas doenças infectoparasitárias de importância na geração de doenças, mas ainda apresentam significantes níveis de prevalência de parasitoses.

No presente estudo podemos observar nas tabelas 2 e 3 que, de maneira geral, os índices de parasitoses vêm diminuindo durante os anos. 0 helminto Schistosoma mansoni foi o que apresentou a maior prevalência. Tem sido relatado que existe uma relação entre baixo nível de escolaridade e esquistossomose, porque o acesso à informação promove a aderência aos cuidados em saúde, mudança comportamental e consequente diminuição dos índices ${ }^{10}$. Desta maneira, podemos perceber a importância da educação em saúde na atenuação da infecção parasitária.

A segunda infecção mais prevalente nesse estudo foi a ascaridíase, sendo mundialmente a parasitose mais frequente. Entretanto, em Jequié a esquistossomose é endêmica, o que justifica sua predominância. Podemos salientar que, nos anos de 2016 e 2017 o helminto A. lumbricoides apresentou maior taxa de prevalência.
O helminto Enterobiusvermicularis teve uma baixa taxa (1,9\%), devido à baixa sensibilidade (apenas de 10\%) do método usado em comparação com ao método de Ghram ou fita gomada ${ }^{11}$.

As práticas educativas, quando bem aplicadas, levam as pessoas a adquirirem os conhecimentos para a prevenção e a redução das enteroparasitoses ${ }^{2}$. Dados obtidos mostram uma substancial redução na prevalência das enteroparasitoses em escolares no Sul do país ${ }^{12}$. E no presente estudo também observamos uma redução das parasitoses nos últimos anos. Desta forma, estes resultados estão de acordo com os encontrados na literatura.

A educação, juntamente com o diagnóstico e tratamento dos parasitados vêm auxiliando os programas de controle da esquistossomose e parasitoses intestinais, principalmente em cidades que são endêmicas para algumas parasitoses, como é o caso do município de Jequié.

\section{Conclusão}

O município de Jequié ainda enfrenta problemas com a esquistossomose devido aos rios contaminados, e uma grande parcela de ribeirinhos que fazem uso dessas águas. Os estudos epidemiológicos relacionados à prevalência das parasitoses intestinais possuem importante função no combate, prevenção e conhecimento a respeito das infecções enteroparasitárias, uma vez que servem como parâmetros para novos estudos.

Além disso, a epidemiologia pode servir como complemento para a prática médica, tendo como base as áreas endêmicas que fornecem informações complementares, auxiliando o diagnóstico clínico das parasitoses intestinais. 
A prevalência das enteroparasitoses vem diminuindo ao longo dos anos. Apesar dos esforços realizados pelo município e estudos como esse, pode-se concluir que ainda precisa melhorar o saneamento básico da periferia e investir mais na educação escolar.

\section{Referências:}

1- Pedraza DF, Queiroz D e Sales MC. Doenças infecciosas em crianças pré-escolares brasileiras assistidas em creches. Ciênc Saúde Coletiva2014;19(2):511-28.

2- Coscrato G, Pina JC e Mello DF. Utilização de atividades lúdicas em educação em saúde: uma revisão interativa da literatura. Acta Paul Enferm2010;23(2):257-63.

3- Belo VS, Oliveira RB, Fernandes PC, Nascimento BWL, Fernandes FV, Castro CLF, et al. Fatores associados à ocorrência de parasitoses intestinais em uma população de crianças e adolescentes. Rev. paul. Pediatr2012;30(2):195201.

4- Rodrigues SR. Projeto Parasitoses Intestinais em crianças: prevalência e fatores associados. Rev. Ciênc. Ext. 2018. v.14,n.3p.50-63.

5- Stephenson L. The impact of schistosomiasis on human nutrition.Parasitology (London) 1993;107(Suppl):S107-23.

6- Vasconcelos IAB, Oliveira JW, Cabral FRF, Coutinho HDM, Menezes IRA.Prevalência de parasitoses intestinais entre crianças de 4-12 anos no Crato, Estado do Ceará: um problema recorrente de saúde pública. Acta Scientiarum Health Sciences2011;v.33,n.1,p.35-41.

7- Schall VT, Jurberg P, Almeida EM, Casz C, Cavalcante FG, Bagno $S$. Educação em saúde para alunos de primeiro grau: avaliação de material para ensino e profilaxia da esquistossomose. Rev. Saúde Pública [Internet]. 1987;21(5):387-404.

8- Hoffman WA, Pons JA, Jan JL. The sedimentation concentrarion method in schistomiasismansoni.Puerto Rico.Journal of Public Health and Tropical Medicine, New York1934.;v.9,p.283-91.

9- Maia, CVA, Hassum, IC. Parasitoses intestinais e aspectos sociossanitários no nordeste brasileiro no século XXI: uma revisão de literatura. Rev. Bras. de Geog. Médica e da Saúde 2016;v.12,N.23p.20-30.
10- Melo AGS, Irmão JJM, Jeraldo VLS, Melo CM. Esquistossomose mansônica em famílias de trabalhadores da pesca de área endêmica de Alagoas. Esc. Anna Nery 2019;23(1)e20180150.

11-Neves, DP. Parasitologia Humana. 13. ed. São Paulo: Atheneu; 2016.

12- Basso RMC, Silva RRT, Soligo DS, Ribacki SI, Callegari-Jacques SM, Zoppas BCA. Evolução da prevalência de parasitoses intestinais em escolares em Caxias do Sul, RS. Rev. Soc. Bras. Med. Trop. [Internet]. 2008;41(3):263-68.

\section{Endereço para Correspondência}

Universidade Federal de Campina Grande- UFCG

R. Aprígio Veloso, 882 - Universitário, Campina Grande - PB

CEP.:58428-830

e-mail: rafaeljan.med@gmail.com

Recebido em 22/11/2019

Aprovado em 13/04/2020

Publicado em 30/03/2020 